\title{
Lattice Properties of Two-Dimensional Charge-Stabilized Colloidal Crystals
}

\author{
Pavel Dyshlovenko ${ }^{1}$ and Yiming $\mathrm{Li}^{2}$ \\ 1 Laboratory of Computer Simulations, Ulyanovsk State technical University, \\ Ulyanovsk 432027, Russia \\ pavel@ulstu.ru \\ 2 Department of Communication Engineering, National Chiao Tung University, \\ Hsinchu 300, Taiwan \\ ymli@faculty.nctu.edu.tw
}

\begin{abstract}
In this paper, electrostatic interaction in two-dimensional colloidal crystals obeying the non-linear Poisson-Boltzmann equation is studied numerically. We first give an overview of the recently developed approach to study of the lattice properties of colloidal crystals. The central point of the theory is determination of the force constants, which are the coefficients of the energy quadratic form of the crystal. Particular attention is given to the symmetry considerations. Some prospective topics of research are briefly discussed.
\end{abstract}

\section{Introduction}

Colloidal crystals are dispersions of colloidal particles arranged into a regular lattice. Besides their importance for studying structural phase transitions resembling conventional melting and freezing, they are also well-defined model systems whose macroscopic properties can be directly connected to the underlying microscopic interparticle interactions.

Theoretical analysis of lattice properties of colloidal crystals, such as normal modes of oscillations or elastic properties, is mostly based on the representation of the potential energy of the particles as a sum of pair interactions. Although the concept of pairwise interaction is adequate for many systems, such as the dipole-dipole interaction [1, 2] and purely entropic forces [3, 4, it fails for chargestabilized colloidal crystals. In particular, it was shown that charge-stabilized colloidal crystals' elastic properties observed cannot be understood within the idea of linear superposition of pairwise interactions [5].

In this paper, we consider a two-dimensional charge-stabilized colloidal crystal obeying the general nonlinear Poisson-Boltzmann (PB) equation. Description of recently proposed approach [6, 7] is included. Our approach enables the force constant determination and successfully provides quantitative estimation of the many-particle effects. It validates the approximation of the nearest neighbor interaction in such systems. In particular, it turns out that the contribution of many-particle to the total electrostatic potential energy is significant and cannot be neglected for a broad range of particle radii and crystal lattice parameters. 
This paper is organized as follows. In Section 2, we state the model formulation. In Section 3, we state the force constants determination. In Section 4, we discuss the role of symmetry. In Section 5, we present the numerical methods. Section 6 reports the results. Section 7 draws conclusions.

\section{Mathematical Model Formulation}

The colloidal crystal under consideration is shown in the Fig. 1. It consists of infinitely long cylindrical colloidal particles of radius $R$ arranged in a twodimensional hexagonal lattice with the lattice constant $a$. The system of the particles is immersed in symmetrical univalent electrolyte. The particles are perfectly rigid dielectric rods. The dielectric permittivity of the particles is much smaller then the one of electrolyte solution, so it is set to be zero for all numerical calculations in the paper. The particles are charged with uniform surface charge density $\sigma$ which is kept constant (the so-called constant-charge or cc-model). The crystal system considered in the paper can be pertinent to the behavior of rodlike objects like DNA molecules, tobacco mosaic viruses [8] and fd viruses [9], rodlike polyelectrolytes [10] or some mesoscopic objects [1]. Throughout the present paper, length and electro-static potential are expressed in units of Debye length $\kappa^{-1}=\left(2 n q_{e}^{2} / \epsilon k T\right)^{-1 / 2}$ and $k T / q_{e}$ respectively, where $n$ is the concentration of either of the species in the electrolyte, $q_{e}$ is the absolute value of the electronic charge, $\epsilon$ is the absolute permittivity of the electrolyte, $k$ is the Boltzmann constant, $T$ is the absolute temperature, and the rationalised SI system of units is used to express the factors.

Electric potential $\phi$ in such a system obeys the non-linear PB equation 12 in the electrolyte's domain outside the particles and the Laplace equation in the interior of the particles:

$$
\frac{\partial^{2} \phi}{\partial x^{2}}+\frac{\partial^{2} \phi}{\partial y^{2}}= \begin{cases}\sinh \phi, & \text { (in the electrolyte) } \\ 0, & \text { (inside the particles) }\end{cases}
$$

Electric field at the surface of the particles meets the electrostatic boundary condition

$$
E_{n}=\sigma,
$$

where $E_{n}$ is a normal component of the electric field in the electrolyte and dielectric permittivity of the particles is equal to zero. As usual, the tangential component of the electric field at the interface remains continuous.

There are no net forces on the particles when all the particles are located in their equilibrium positions. If one or more of the particles are displaced from the equilibrium, the non-zero net forces on them arise. The force on any particle in the system can be calculated by means of integration of the stress tensor:

$$
\mathbf{F}=\oint_{\Gamma}\left[\nabla \phi \otimes \nabla \phi-\left(\frac{1}{2}|\nabla \phi|^{2}+\cosh \phi-1\right) I\right] \cdot \mathbf{n} d \Gamma,
$$




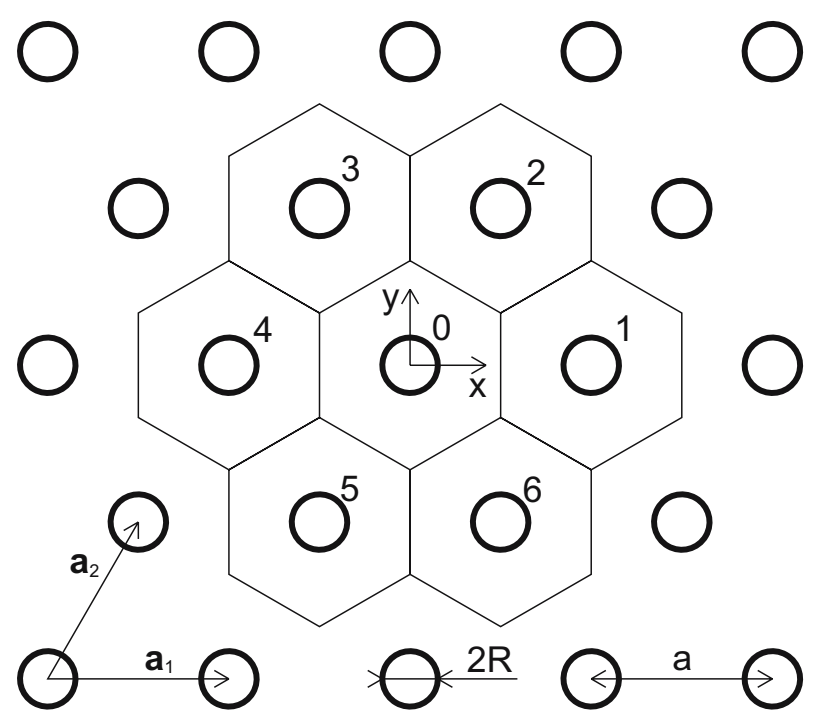

Fig. 1. Two-dimensional hexagonal colloidal crystal lattice. Particles are infinite rods perpendicular to the plane of the figure. Domain of the numerical problem comprises seven particles 0 to 6 with corresponding hexagonal Wigner-Seitz cells.

where $\Gamma$ is a Wigner-Seitz cell's boundary, $\mathbf{n}$ is an outer normal vector to the element $d \Gamma$, and $I$ is an identity matrix. Although any closed loop enclosing the particle can be employed for the integration, using of the boundary of the Wigner-Seitz cell is practical since it provides rather low errors in process of numerical implementation of the integration.

\section{Force Constants Determination}

Classical potential energy $V$ of a crystal for small displacements of the particles from the equilibrium positions can be written [13] as follows:

$$
V=\frac{1}{2} \sum_{\alpha, \beta, \mathbf{N}, \mathbf{M}}\left(\frac{\partial^{2} V}{\partial Z_{\alpha, \mathbf{N}} \partial Z_{\beta, \mathbf{N}+\mathbf{M}}}\right) Z_{\alpha, \mathbf{N}} Z_{\beta, \mathbf{N}+\mathbf{M}}
$$

where $Z_{\alpha, \mathbf{N}}$ is an $\alpha$-component of the displacement $Z$ from the equilibrium position pointed by vector $\mathbf{N}, \alpha=x, y, \beta=x, y, \mathbf{N}$ and $\mathbf{M}$ are vectors of the Bravais lattice. Coefficients

$$
C_{\alpha \beta}^{\mathbf{M}}=\frac{\partial^{2} V}{\partial Z_{\alpha, \mathbf{N}} \partial Z_{\beta, \mathbf{N}+\mathbf{M}}}
$$

of the quadratic form (4) are called force constants. They do not depend on $\mathbf{N}$ and can be arranged into the square matrix $C=\left\|C_{\alpha \beta}^{\mathrm{M}}\right\|$ of a quadratic form, 
the coefficients $C_{\alpha \beta}^{\mathbf{M}}$ at fixed $\mathbf{M}$ constituting the $2 \times 2$ submatrix $C^{\mathbf{M}}$ of the general matrix, and $\mathbf{M} \in\{(0,0),(1,0),(0,1),(-1,1),(-1,0),(0,-1),(1,-1)\}$ in our consideration.

Determination of the force constants is based on the observation that the first derivatives $\left(\partial V / \partial Z_{\alpha, \mathbf{N}}\right), \alpha=x, y$, are merely the components of the force on the particle $\mathbf{N}$. The forces can be calculated directly by integrating the stress tensor, as it has been mentioned above, after the solution of the PB equation has been obtained. Then, the primary numerical data for the forces on the particles of the system exerted by the shift of the central particle 0 are transformed into the forces $F_{\alpha, 0}$ on the central particle caused by the shifts of different particles using the symmetries of the crystal. These forces are expressed as the functions of corresponding displacements, both positive and negative: $F_{\alpha, \mathbf{0}}=F_{\alpha, \mathbf{0}}\left(Z_{\beta, \mathbf{M}}\right)$, $\alpha=x, y, \beta=x, y, \mathbf{M} \in\{(0,0),(1,0),(0,1),(-1,1),(-1,0),(0,-1),(1,-1)\}$. Finally, the force constants are obtained as $C_{\alpha \beta}^{\mathrm{M}}=-\partial F_{\alpha, \mathbf{0}} / \partial Z_{\beta, \mathbf{M}}$. The differentiation was carried out by fitting the numerical data for the functions $F_{\alpha, \mathbf{0}}=F_{\alpha, \mathbf{0}}\left(Z_{\beta, \mathbf{M}}\right)$ with polynomials of power 7 and taking the coefficient of the linear term as the first derivative at point 0 .

\section{Role of Symmetry}

Taking into account the symmetry of the crystal enables significant reduction of the amount of numerical calculations. First, direct calculation of the forces on the central particle 0 would require multiple solutions of the PB equation for configurations with different particles shifted from their equilibrium positions. Translational and inversion symmetry of the crystal lattice makes it possible to reduce the calculation of the forces on the central particle 0 arising from the motion of different particles to the calculation of the forces on all the particles (seven here) due to the motion of only the central particle alone. Having the numerical solution of the PB equation for the configuration with only the central particle shifted, the forces on all the particles in the system can then be obtained by taking the integral of the stress tensor over the corresponding contours. The post-solution integration is much less expensive in the sense of computer resources required than the numerical solution itself.

Second, mirror symmetry of the problem allows the use of only a half of the problem's domain. When the particle 0 is shifted along the x-axis, the problem retains the mirror symmetry about this axis. Thus, without loss of generality, the upper half of the domain above the $\mathrm{x}$-axis is required.

Finally, rotational symmetry of the crystal lattice allows further reduction of the calculations at the post-solution stage. The seven particles under consideration belong to two different orbits of the rotational subgroup of the crystal point group. The first orbit consists of only the central particle 0 . The particles 1 to 6 constitute another orbit: they transform into each other when rotating about the point 0 at the angle multiple of $\pi / 3$. For symmetry reasons, matrix $C^{(0,0)}$ has diagonal elements equal to each other and off-diagonal ones equal to zero. Consequently, matrix $C^{(0,0)}$ is completely determined by only one, say $C_{x, x}^{(0,0)}$, of 
its diagonal element. For the same reasons, matrix $C^{(1,0)}$ for the particle 1 has zero off-diagonal elements and is thus determined by only two diagonal elements $C_{x, x}^{(1,0)}$ and $C_{y, y}^{(1,0)}$. Since particles 1 to 6 belong to the same orbit of the rotational subgroup of the crystal point group the force constant matrices of these particles are not independent. If the matrix of the particle described by vector $M$ is known, the matrices of the other particles can be obtained by the matrix transformation according to the rule of quadratic forms' matrix transformation [9]:

$$
C^{\mathbf{N}}=R^{\mathrm{T}}(\phi) C^{\mathrm{M}} R(\phi),
$$

where $\mathbf{M}, \mathbf{N} \in\{(1,0),(0,1),(-1,1),(-1,0),(0,-1),(1,-1)\}, \phi$ is the angle between the vectors $\mathbf{N}$ and $\mathbf{M}$, and superscript $\mathrm{T}$ means matrix transposition. Therefore, the complete set of 28 force constants $(7$ particles $\times 4$ matrix elements, in the approximation of nearest neighbour interaction) is completely determined only by the three non-trivial independent parameters which should be obtained directly from computer experiments. The other constants are obtained then by means of symmetry transformations (6).

\section{$5 \quad$ Numerical Procedures}

Equations (11) and (2) are solved numerically using the method described in [14,15]. This method combines the finite-element solution of the equation with an adaptive mesh refinement [16, 17, 18. It is well suited for the two-dimensional problems with complicated geometry and variety of boundary conditions. The domain of the problem for numerical solution consists of the Wigner-Seitz cells of the central particle 0 and its six nearest neighbours 1 to 6 . The standard von Neumann boundary conditions hold at the outer boundary of the domain.

The numerical calculations are carried out for the central particle shifted by ten equal steps along the positive direction of the $\mathrm{x}$-axis so that the largest shift amounted to $10 \%$ of the separation distance between the neatest particles. The forces exerted by this shift on all the seven particles on the particles were calculated by means of numerical integration of the stress tensor, as it was described above. Since the domain of the problem is restricted to the seven particles, the interaction of the central particles with its nearest neighbours is only considered in this paper. It is shown in [6] that this is a very good approximation for a broad range of parameters a and $R$.

Domain of the problem with the mesh of triangular elements on it is shown in the Fig. 2, Due to the mirror symmetry about the x-axis only the upper half of the domain is required. The mesh is a Delaunay triangulation of the domain at each stage of the solution.

\section{Results and Discussion}

The force constants of the two-dimensional hexagonal charge-stabilized colloidal crystal for the typical set of parameters $\sigma=2.0, R=1.0$ and $a=5.0$ are shown in Table 1. Only the three independent force constants are presented; the other 


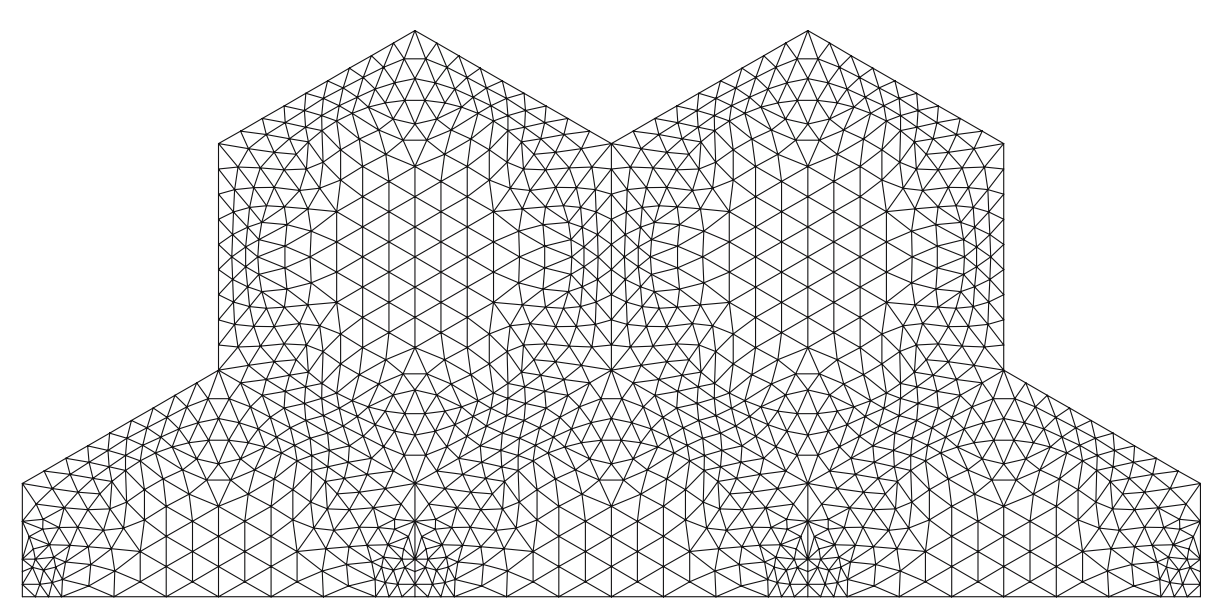

Fig. 2. Domain of the problem and irregular mesh of triangular elements on it. The round boundaries of colloidal particles are clearly observed. The mesh is obtained in the beginning of the solution after the first iteration of the numerical procedure and consists of 2160 triangular elements. The final mesh is obtained after ten iterations comprised more than 135000 elements, concentrated mostly at the outer boundaries of the particles.

ones can be obtained my means of the transformation (6). Complete set of the results and their systematic analysis are provided in [7]. Nevertheless, the example in Table 1 illustrates the main features of the data obtained. First, it turns out that the force constant $C_{y, y}^{(1,0)}$ for the system considered is definitely nonzero, while the theory of harmonic crystal based on the assumption of pairwise interaction between particles requires always this constant to be strictly equal to zero. A quantitative estimation of the contribution of the many-particle interaction into a total electrostatic interaction in a system was introduced in 6]. It was shown there that the collective electrostatic interaction in two-dimensional colloidal crystals cannot be expressed as a sum of pair interactions between the particles and that the many-particle interactions are strong enough for a broad range of charge densities $\sigma$ on the particles, radii $R$ of the particles and lattice parameters $a$ even at very large ones when the interparticle interaction itself is weak. Another feature of the data in Table 1 is that the ratio $C_{x, x}^{(0,0)} / C_{x, x}^{(1,0)}$ is not exactly equal to -3 as it should be according to the approximation of the nearest-neighbor interaction. A quantitative measure of the validity of the nearest-neighbor interaction approximation based on this discrepancy was pro-

Table 1. Force constants of the crystal for $\sigma=2.0, R=1.0$ and $a=5.0$

\begin{tabular}{ccc}
\hline$C_{x, x}^{(0,0)}$ & $C_{x, x}^{(1,0)}$ & $C_{y, y}^{(1,0)}$ \\
\hline 0.6756891 & -0.2663590 & 0.0480621 \\
\hline
\end{tabular}


posed in [6]. It was shown there, that this approximation remains a rather good one for a broad range of crystal parameters $\sigma, R$ and $a$.

\section{Conclusions}

In this paper, we have computationally explored the electrostatic interaction in two-dimensional colloidal crystals. The non-linear Poisson-Boltzmann equation has been solved numerically with adaptive finite element method. An overview of the recently developed approach to study of the lattice properties of colloidal crystals has been reported. The central point of the theory focuses on determination of the force constants, which are the coefficients of the energy quadratic form of the crystal. Particular attention has been given to the symmetry considerations. Some prospective topics of research have briefly been discussed. The characteristics of the crystal system considered in the paper seem to be valid for broader range of the colloidal systems. In particular, the crystal with the particles obeying the constant potential model ( $c p$-model) or recently proposed constant total charge model (ctc-model) should be investigated. Corresponding calculations are currently in progress. Different types of crystal lattices, primarily the square one, are also of interest. One more question to study is the contribution of the neighbors further then the nearest ones into the total electrostatic interaction and corresponding modification of the crystal's properties. To study this problem, further development of the program code is needed to enable sufficient number of particles in the system. There are no doubts that three-dimensional colloidal crystals possess many features of their two-dimensional counterparts. However, study of three-dimensional problems will require efforts for new program code development utilizing the power of modern libraries for partial differential equation solution and involving some kind of parallelization [19].

\section{Acknowledgments}

This work was supported in part by Taiwan National Science Council (NSC) under Contract NSC-94-2215-E-009-084 and Contract NSC-95-2752-E-009-003PAE, by the Ministry of Economic Affairs, Taiwan under Contract 93-EC-17-A07-S1-0011, and by the Taiwan semiconductor manufacturing company under a 2005-2006 grant. One of the author (P.D.) gratefully acknowledges the financial support from the Mianowski Fund of Foundation for Polish Science during his visit to the Institute of Catalysis and Surface Chemistry (Cracow) where a part of the present work was carried out.

\section{References}

1. Keim, P., Maret, G., Herz, U., von Grünberg, H.H.: Harmonic lattice behavior of two-dimensional colloidal crystals. Phys. Rev. Lett. 92 (2004) 215504

2. Hay, M.B., Workman, R.K., Manne, S.: Two-dimensional condensed phases from particles with tunable interactions. Phys. Rev. E 67 (2003) 012401 
3. Cheng, Z., Zhu, J., Russel, W.B., Chaikin, P.M.: Phonons in an entropic crystal. Phys. Rev. Lett. 85(7) (2000) 1460-1463

4. Penciu, R.S., Kafesaki, M., Fytas, G., Economou, E.N., Steffen, W., Hollingsworth, A., Russel, W.B.: Phonons in colloidal crystals. Europhys. Lett. 58(5) (2002) 699704

5. Weiss, J.A., Larsen, A.E., Grier, D.G.: Interactions, dynamics, and elasticity in charge-stabilized colloidal crystals. J. Chem. Phys. 109(19) (1998) 8659-8666

6. Dyshlovenko, P.E.: Evidence of many-particle interactions in two-dimensional charge-stabilized colloidal crystals. Phys. Rev. Lett. 95 (2005) 038302

7. Dyshlovenko, P.E. (the paper in preparation)

8. Adams, M., Fraden, S. Biophys. J. 74 (1998) 669

9. Purdy, K.R., Dogic, Z., Fraden, S., Rühm, A., Lurio, L., Mochrie, S.G.J.: Measuring the nematic order of suspensions of colloidal fd virus by x-ray diffraction and optical birefringence. Phys. Rev. E 67 (2003) 031708

10. Guilleaume, B., Blaul, J., Ballauff, M., Wittemann, M., Rehahn, M., Goerigk, G.: The distribution of counterions around synthetic rod-like polyelectrolytes in solution. Eur. Phys. J. E 8 (2002) 299-309

11. de A. A. Soler-Illia, G.J., Sanchez, C., Lebeau, B., Patarin, J.: Chemical strategies to design textured materials: from microporous and mesoporous oxides to nanonetworks and hierarchical structures. Chem. Rev. 102 (2002) 4093-4138

12. Israelachvili, J.N.: Chap. 12. In: Intermolecular and Surface Forces. Academic Press (1991)

13. Feynman, R.P.: Chap. 1. In: Statistical Mechanics. W. A. Benjamin, Inc., Massachusetts (1972)

14. Dyshlovenko, P.E.: Adaptive mesh enrichment for the poissonboltzmann equation. J. Comp. Phys. 172 (2001) 198-208

15. Dyshlovenko, P.E.: Adaptive numerical method for poissonboltzmann equation and its application. Comp. Phys. Commun. 147 (2002) 335-338

16. Li, Y., Sze, S.M., Chao, T.S.: A Practical Implementation of Parallel Dynamic Load Balancing for Adaptive Computing in VLSI Device Simulation. Comp. Phys. Commun. 147 (2002) 335-338

17. Li, Y., Chao, T.S., Sze, S.M.: A Domain Partition Approach to Parallel Adaptive Simulation of Dynamic Threshold Voltage MOSFET. Eng. Comput. 18 (2002) 124-137

18. Li, Y., Yu, S.M.: A Parallel Adaptive Finite Volume Method for Nanoscale Doublegate MOSFETs Simulation. J. Comput. Appl. Math. 175 (2005) 87-99

19. Li, Y.: A Parallel Monotone Iterative Method for the Numerical Solution of Multidimensional Semiconductor Poisson Equation. Comp. Phys. Commun. 153 (2003) $359-372$ 